Condiciones de calidad de vida en el trabajo de las mujeres del sector agropecuario. Cantón Rumiñahui.

Saskia García Aucatoma, Narciza Paredes, Ligia García Rosero. 


\title{
Condiciones de calidad de vida en el trabajo de las mujeres del sector agropecuario. Cantón Rumiñahui.
}

\section{Conditions of quality of life in the work of women in the agricultural sector. Cantón Rumiñahui.}

\author{
Saskia García Aucatoma, Narciza Paredes, Ligia García Rosero \\ saskia.garcia@gmail.com
}

\begin{abstract}
Resumen
Se analizaron las condiciones de calidad de vida del trabajo para las mujeres del sector agropecuario en el Cantón Rumiñahui; para ello se realizó una caracterización socio-económica, luego se identificaron las dimensiones de la calidad de vida en el trabajo obteniéndose una valoración objetiva y subjetiva de sus dimensiones. Se utilizaron técnicas cuantitativas y cualitativas y se aplicaron pruebas estadísticas (Alfa de Cron Bach, prueba de U de Mann-Whitney y la prueba de Kruskal Wallis) para la validación de los resultados. Se tomó una muestra 84 trabajadoras agropecuarias. Se determinó que el $50 \%$ cuenta con educación primaria, un $50 \%$ proveen económicamente sus hogares, $71 \%$ no poseen afiliación al seguro social, y el $20 \%$ desconoce los peligros a los que están expuestas en su trabajo. La Calidad de Vida en el trabajo, es percibida como baja, y los factores determinantes son la falta de contratación y regularización laboral, la carencia de salud y seguridad social, la exposición a riesgos en el trabajo y la baja remuneración.
\end{abstract}

Palabras clave: Calidad de vida en el trabajo, seguridad en el trabajo, mujeres.

\begin{abstract}
Quality of work conditions for women in the agricultural sector were analyzed in the Rumiñahui Canton; for this purpose a socio-economic characterization was carried out, then the dimensions of the quality of life in the work were identified, obtaining an objective and subjective assessment of its dimensions. Quantitative and qualitative techniques were used and statistical tests (Cron bach's alpha, Mann-Whitney $U$ test and Kruskal-Wallis test) were used for the validation of the results. A sample of 84 agricultural workers was taken. It was determined that 50\% have primary education, $50 \%$ provide their homes economically, $71 \%$ do not have social security affiliation, and $20 \%$ do not know the dangers to which they are exposed in their work. The quality of life at work is perceived as low, and the determining factors are the lack of labor recruitment and regularization, lack of health and social security, exposure to risks at work and low remuneration.
\end{abstract}

Keywords: Quality of life at work, safety at work, women.

\section{Introducción}

Desde sus inicios, la economía del desarrollo se ha ocupado fundamentalmente del enriquecimiento material, esto es el incremento del volumen de producción de bienes y servicios. (Keith, 2001). El nexo entre mayor producto y menor pobreza se consideraba tan fuerte que muchos economistas estaban convencidos de que el crecimiento se convertía no sólo en el medio para alcanzar el desarrollo sino en el fin del desarrollo mismo. Y aunque ciertamente siempre hubo disidentes, estos más que cuestionar la prioridad del crecimiento como tal han tendido a matizarla resaltando la importancia de la distribución de los beneficios generados por el crecimiento. La mayoría de los debates en torno a posibles estrategias alternativas de desarrollo no iban más allá de discutir la mejor forma de acelerar el incremento de la producción de bienes y servicios (Griffin, 2001).

El Desarrollo Humano (DH) es una forma de medir la calidad de vida del ser humano en el 
medio en que se desenvuelve (Contreras, 2014). El DH es el resultado de la interacción de muchos factores y condicionantes económicas y sociales, las que actuando de manera dinámica, en los contextos culturales particulares de los pueblos, imprimen su sello característico a las sociedades (Reyes, 2009). El trabajo de Sen, premio Nobel en 1998, fue fundamental para impulsar la agenda intelectual y política del desarrollo humano, al redefinir el desarrollo como aumento de las oportunidades de las personas y desarrollo de sus capacidades (Sen, 1992).

Por otra parte, las estadísticas de la Organización Internacional del Trabajo (OIT) indican que las condiciones de trabajo para la mayoría de los trabajadores en todo el mundo no reúnen los estándares y guías mínimos respecto salud ocupacional, seguridad en el trabajo, protección social y calidad de vida laboral del trabajador (Pérez et al. 2011).

Pese a ello, es necesario tener en cuenta que los cambios que suceden en los sistemas agrícolas en torno al trabajo y las organizaciones circundantes (por ejemplo, prolongadas e intensas jornadas de trabajo, sobrecarga de trabajo, mayores exigencias cognitivas y emocionales, inseguridad laboral, contratos injustos, etc.) rebasan la postura de los modelos tradicionales de prevención y protección de la salud ocupacional, que se perfilaban más bien desde una postura clínica y centrados en el daño o la enfermedad. En este sentido, la psicología de la salud ocupacional se enfoca en la aplicación de la psicología para mejorar la calidad de vida en el trabajo y para proteger y promover la seguridad, la salud y el bienestar de los trabajadores (Pérez, 2013).

En el Ecuador, el tema de la calidad de vida en el trabajo, aún no es considerado como una prioridad y aún menos en el sector agropecuario. Por ello, este estudio está dirigido a las trabajadoras mujeres de la sociedad que desempeñan un papel fundamental como trabajadoras y en otras facetas como madres de familia con una doble carga de trabajo.

El rápido crecimiento que han tenido a lo largo de los últimos años las explotaciones agropecuarias tanto en la región como en el Ecuador es significativo, encontrándose en la mano de obra femenina una tendencia a la feminización del trabajo. La argumentación empresarial está relacionada con la afirmación de que las mujeres son más delicadas y cuidadosas. Pero en realidad las mujeres son socialmente más vulnerables. Por ser muchas de ellas jefas de hogar, se someten a cualquier trabajo en cualquier condición, con tal de garantizar un ingreso para su familia. Así, la mano de obra femenina se encuentra en una situación laboral entre lo invisible, lo precario y lo desigual (Osorio, 2014). Esto se suma, como ya se ha visto, a que en general la pobreza se acentúa más en las mujeres.

La presente investigación tuvo como objetivo realizar una caracterización socio económica enfocada a las condiciones de calidad de vida del trabajo para las mujeres del sector agropecuario en el Cantón Rumiñahui, Provincia de PichinchaEcuador; luego, para el mismo grupo, se identificaron las dimensiones de la calidad de vida en el trabajo para una concluyente valoración objetiva y subjetiva de las mismas.

\section{Materiales y métodos}

La investigación se realizó acorde a lo sugerido por Pérez (2013), para quien, lo adecuado para este tipo de estudios es un enfoque mixto que combine un estudio cualitativo y cuantitativo cuya finalidad será determinar el efecto del trabajo en la Calidad de Vida. Se utilizaron variables sociodemográficas de género, nivel de educación puesto de trabajo, horario, beneficios extras, etc.

Este proyecto está basado en la investigación bibliográfica, se han revisado bases de datos, bibliotecas virtuales, libros para su análisis e interpretación con el propósito de conocer las contribuciones científicas del pasado y establecer relaciones y diferencias con el estado del conocimiento actual.

El trabajo de campo se realizó mediante visitas de las casas de las trabajadoras rurales informales y en sus lugares de trabajo (explotaciones agropecuarias), se realizó a través del contacto directo del investigador con la realidad. Se recolectaron y registraron datos y toda la información posible de manera ordenada. Las herramientas de investigación se basaron en información primaria en la que se consideraron entrevistas usando preguntas elaboradas. Las entrevistas permitieron recolectar datos cuantitativos sobre el tema de las condiciones de trabajo por medio de preguntas cerradas y abiertas.

Esta herramienta permitió obtener información de la realidad laboral en el sector agropecuario del Cantón Rumiñahui. La información secundaria es referida a la investigación de datos $\mathrm{y}$ estudios obtenidos por terceros, instituciones gubernamentales, entre otros.

Como parte del análisis de la información relevada en la información primaria se elaboró la tabulación de datos mediante la aplicación de la estadística descriptiva tanto como para tabular 
como para graficar los datos recolectados usando el programa estadístico SPSS.

Construir un instrumento válido y confiable para medir la Calidad de Vida de las Trabajadoras en el contexto local disminuyó la variabilidad del método de medición. Para asegurar la fortaleza del instrumento, se determinó su viabilidad, confiabilidad y validez; la viabilidad se aseguró mediante el empleo de un cuestionario donde se cuida el tiempo empleado para su cumplimentación, la sencillez y la amenidad del formato, el interés, brevedad y claridad de las preguntas, así como la corrección, interpretación y presentación de los resultados. La confiabilidad, entendida como el grado en que su aplicación repetida al mismo sujeto $\mathrm{u}$ objeto produce resultados iguales, y la validez, referida como el grado en que un instrumento realmente mide la variable que pretende medir (Peñarrieta et. al. 2014).

Así, luego de la revisión de las variables se determinó trabajar con cinco dimensiones: seguridad en el trabajo, satisfacción por el trabajo, bienestar logrado a través del trabajo, desarrollo personal, administración del tiempo libre.

Se realizó una investigación descriptiva por medio de la técnica de la encuesta aplicada a una muestra de las mujeres trabajadoras del sector agropecuario en el Cantón Rumiñahui; se caracterizó la realidad respecto las condiciones de trabajo. Con respecto al muestreo se identificó que el más apropiado es el muestreo probabilístico.

\section{Prueba piloto}

Para asegurar la calidad de los datos y la calibración de las herramientas de diagnóstico a usarse e realizó una prueba piloto bajo las siguientes características: 1. La prueba se realizó a 10 personas; 2. Considerar una pregunta filtro para obtener las variables p y q; 3. Realizar preguntas con contenido, comprensible, amigable, preciso.

El cuestionario se aplicó a 10 mujeres del sector agropecuario. Este pre test permitió identificar:

- Tipos de preguntas más adecuados.

- Si el enunciado es correcto y comprensible, y si las preguntas tienen la extensión adecuada.

- Si es correcta la categorización de las respuestas.

- Si existen resistencias psicológicas o rechazo hacia algunas preguntas.
- Si el ordenamiento interno es lógico; si la duración está dentro de lo aceptable por los encuestados.

En cuanto a los métodos utilizados para la realización del pre test cognitivo, éste se llevó a cabo mediante la realización de: entrevistas informales, grupos focales de la población diana, encuesta sobre comprensión de las preguntas, valoración del cuestionario por parte de los participantes en el estudio.

El resultado de Alfa de Cron Bach es de 0.959, mismo que confirmó la fiabilidad del instrumento.

\section{Validación del instrumento}

Luego de realizar la prueba piloto se realizó una validación del instrumento y se obtuvieron las siguientes conclusiones: La dimensión de soporte institucional no se valora en este estudio debido a que las subdimensiones miden el proceso de trabajo, la supervisión laboral, el apoyo de los superiores, la evaluación del trabajo, la oportunidad de promoción y autonomía siendo todos estos dependientes de un empleo formal, en el caso del sector agropecuario esto no se puede aplicar ya que en la prueba piloto inicial la mayoría de trabajadoras no contaban con empleo, y sobre todo valora procedimientos de trabajo que son inexistentes en el grupo de estudio.

La dimensión de integración al puesto de trabajo no fue valorada por no ser pertinente ni apropiada al grupo de estudio dirigido, las subdimensiones miden pertinencia, motivación y ambiente de trabajo, en el sentido de empresa y preparación académica, criterios que el grupo de estudio carece.

De la dimensión de Administración del tiempo libre se elimina la subdimensión planificación del tiempo libre, analiza el trabajo con el criterio de si es excesivo, se lleva a la casa en el sector agropecuario esto no es dable.

\section{Población y tamaño de la muestra}

La fórmula para la obtención del tamaño de la muestra para el presente proyecto será para población finita, siendo la siguiente:

\section{FÓRMULA PARA OBTENER LA MUESTRA.}

$$
\begin{aligned}
\mathrm{n}= & \mathrm{z} 2 \cdot \mathrm{p} \cdot \mathrm{q} \cdot \mathrm{N} \\
& \mathrm{e} 2 \cdot(\mathrm{N}-1)+\mathrm{z} 2 \cdot \mathrm{p} \cdot \mathrm{q} .
\end{aligned}
$$

$\mathrm{N}=$ Universo 700

$\mathrm{P}=$ Casos a favor 0.50 
$\mathrm{Q}=$ Casos en contra 0.50

$\mathrm{E}=$ Error 0.10

$\mathrm{Z}=$ Nivel de Confianza 1.96

$$
n=\frac{1.962 \times 700 \times 0.50 \times 0.50}{(0.10) 2(700-1)+(1.96) 2 \times 0.50 \times 0.50}
$$

$\mathrm{n}=85$

\section{Entrevistas}

Para el trabajo de campo se consideraron todas las preguntas o dudas presentadas por las mujeres trabajadoras. La metodología de campo consistió en aplicar la encuesta el día de mayor concurrencia de trabajadoras a la feria de ventas de sus productos en el mercado central de la principal ciudad de estudio que fue Sangolquí.

En la feria se recolectaron las encuestas aplicándolas de forma individual, previa una explicación de los objetivos de la misma y del método a aplicar, no se presentaron dudas de fondo ni forma. Se dividió por sectores de aplicación entre los que se mencionan: área pecuaria, venta de productos cárnicos, pollos, porcinos. Frutas, vegetales, plantas, semillas, verduras, granos, papas.

Se viajó a los centros de producción de maíz, habas, ganado vacuno, leche, especies menores y se aplicó la encuesta. Se conversó con las líderes del sector quienes orientaron y apoyaron en el proceso de la aplicación de la encuesta.

Se visitaron los centros de producción de maíz, habas, ganado vacuno, leche, especies menores y se aplicó la encuesta. Se conversó con las líderes del sector quienes orientaron y apoyaron en el proceso de la aplicación de la encuesta.

\section{RESULTADOS Y DISCUSIONES}

\section{Análisis de Fiabilidad de la Escala del Instrumento}

Acorde a los resultados del Cuadro 1, donde se representa el análisis de fiabilidad para el instrumento aplicado usando una muestra de 84 encuestas con 23 preguntas de satisfacción de Calidad de Vida en el Trabajo de las mujeres del sector agropecuario del Cantón Rumiñahui, el valor Alfa de Cron Bach es de 0.924, lo que significa que, el instrumento aplicado tiene una excelente consistencia interna entre las variables estudiadas acorde al criterio general de George y Mallery (2003), demostrándose una alta fiabilidad de los resultados de la presente investigación.

Cuadro 1 - Análisis de fiabilidad del instrumento aplicado.

\section{Fiabilidad}

\section{Escala: ALL VARIABLES}

Resumen de procesamiento de casos

\begin{tabular}{|cl|r|r|}
\hline & & \multicolumn{1}{|c|}{ N } & \multicolumn{1}{c|}{$\%$} \\
\hline Casos & Válido & 84 & 90,3 \\
& Excluido $^{\text {a }}$ & 9 & 9,7 \\
& Total & 93 & 100,0 \\
\hline
\end{tabular}

a. La eliminación por lista se basa en todas las variables del procedimiento.

Estadisticas de fiabilidac

\begin{tabular}{|r|r|}
\hline $\begin{array}{c}\text { Alfa de } \\
\text { Cronbach }\end{array}$ & $\begin{array}{c}\text { Nde } \\
\text { elementos }\end{array}$ \\
\hline 924 & 23 \\
\hline
\end{tabular}

En el Cuadro 2, se identifica la fiabilidad de la escala al instrumento aplicado, resultando un valor de 0,924 , confiriendo validez interna a las dimensiones lo que permite aplicar a toda la muestra el cuestionario de manera segura.

Cuadro 2- Análisis de la fiabilidad de escala del instrumento

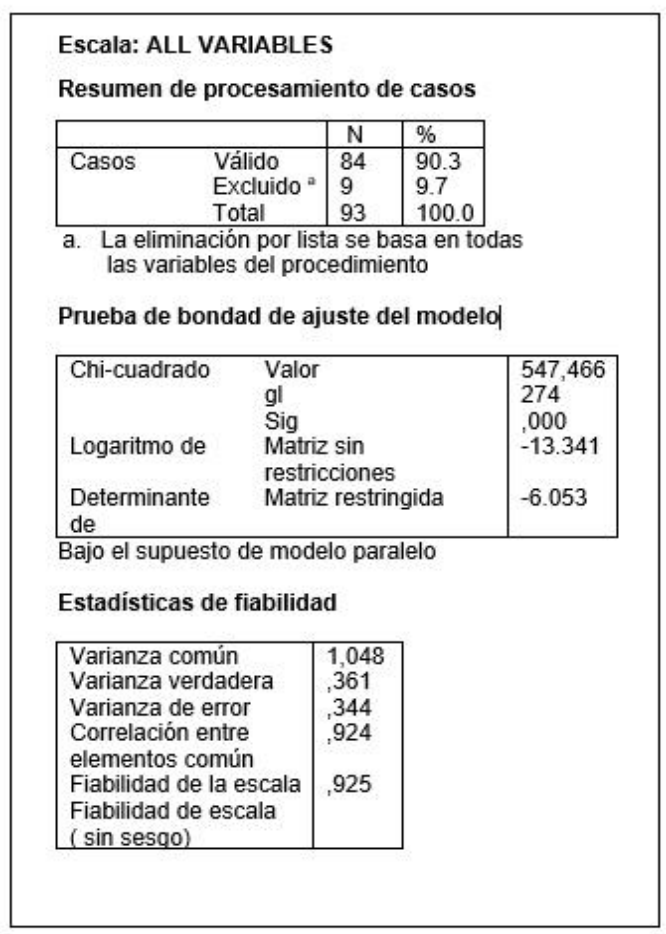

Así, los resultados consolidan una vez más la validez transnacional del instrumento, que ha permitido asumir que los ítems (medidos en 
escala tipo Likert) miden un mismo constructo o dimensión teórica y que están altamente correlacionados (Welch \& Comer, 1988)

\section{Caracterización Socioeconómica}

Se muestra a continuación, un análisis de los resultados por cada ítem general del diagnóstico socioeconómico de las mujeres involucradas con el sector agropecuario en el Cantón Rumiñahui:

Sector donde Labora. - Respecto a la pregunta ¿En qué área usted se encuentra trabajando? (Figura 1), los resultados obtenidos evidencian que un $50 \%$ de las mujeres trabajan en el área de Agricultura, el $27,4 \%$ se dedica al comercio agrícola, el 13,1 \% a Ganadería, al área pecuaria el $7,1 \%$ y son amas de casa el $2,4 \%$. Como conclusión se destaca un grupo importante de trabajadoras dedicadas al comercio agrícola con un $27,4 \%$.

Según el Ministerio de Agricultura Ganadería Acuicultura y Pesca, el 71,9\% de los agricultores se ven forzados a realizar otras labores, únicamente el $28,8 \%$ de los agricultores (medianos y grandes propietarios) dicen sobrevivir con los ingresos provenientes de la actividad agrícola.

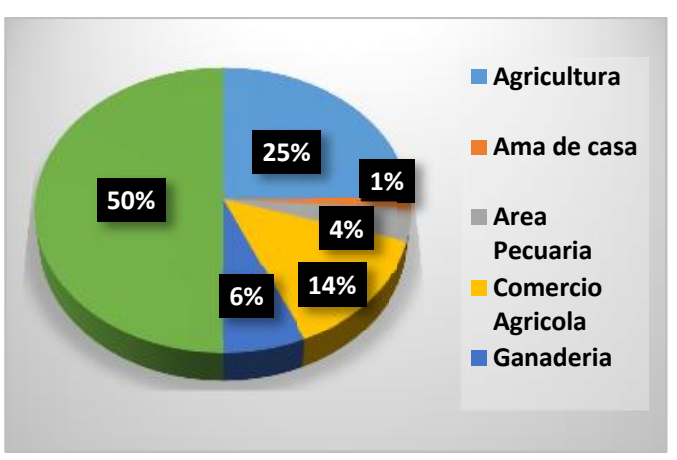

Figura 1. Sector donde labora

Trabajo remunerado. - Los resultados de la figura 2 muestran que el $69 \%$ recibe una remuneración económica o percibe un ingreso por su trabajo, y un $31 \%$ no recibe una remuneración por el trabajo que realiza. No existe formalidad en el empleo y en ocasiones se trabaja para recuperar la inversión inicial y obtener algo de ganancia mediante los productos que venden.

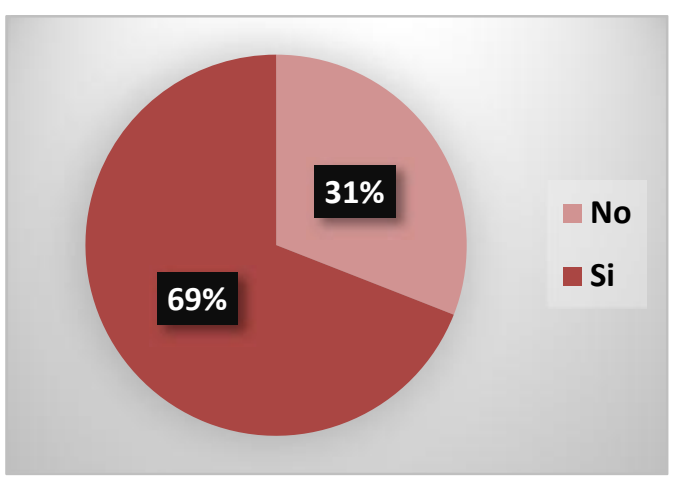

Figura 2.- Trabajo remunerado

Datos del INEC (2016) sobre empleo inadecuado, para septiembre del 2015 se muestra que la tasa es del 49,21\%. Este indicador refiere la realidad del grupo de estudio que trabaja y no recibe paga por su labor formando parte de los trabajadores informales.

Principal proveedor económico en la familia. La figura 3, refleja que el principal proveedor económico en las familias involucradas con el sector agropecuario en el Cantón Rumiñahui, lo comparten en igual porcentaje el padre y la madre, con el $48 \%$ de representación para cada uno. En el 3\% de los casos, los hijos son los principales proveedores del sustento económico en la familia. Esto evidencia el paulatino incremento del rol económico de las mujeres en la familia, convirtiéndose en muchos casos, en la responsable de la generación de ingresos del hogar.

En los últimos años las cifras a nivel nacional han cambiado respecto quien es el proveedor del hogar, el papel de la mujer se ha ampliado, su rol y espacio en el mundo laboral se ha habilitado por sus características y competencias (García, 2015).

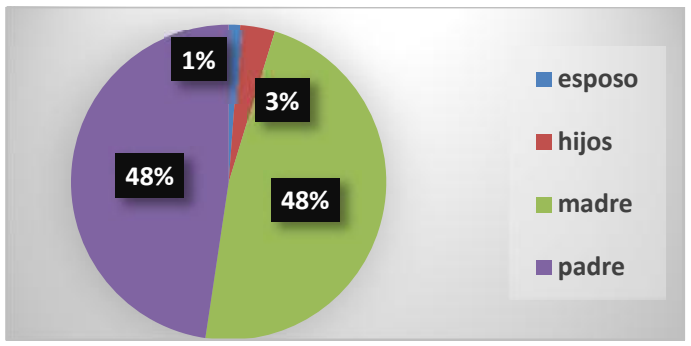

Figura 3.- principal proveedor económico

Se demuestra entonces que el sector Agropecuario cumple con lo señalado por el INEC (2010), que en el Artículo 11 de la Constitución de la República del Ecuador, se reconoce el Principio de Igualdad y no Discriminación el cual establece que "Todas las personas son iguales y gozarán de los mismos derechos, deberes y oportunidades"; por lo tanto el Estado adoptará medidas de acción afirmativa 
que promuevan la igualdad real en favor de los titulares de derechos que se encuentren en situación de desigualdad.

Nivel de educación. - Ante la pregunta: ¿Cuál es su nivel de educación?, la figura 4 muestra que un alto número de mujeres tiene una escolaridad baja siendo muy difícil para las mujeres salir adelante por este factor la falta de educación. El $50 \%$ tiene educación primaria, el $33.3 \%$ tiene educación secundaria, $1.1 \%$ tienen educación técnica, $2.2 \%$ educación superior y el $4.3 \%$ ningún tipo de educación.

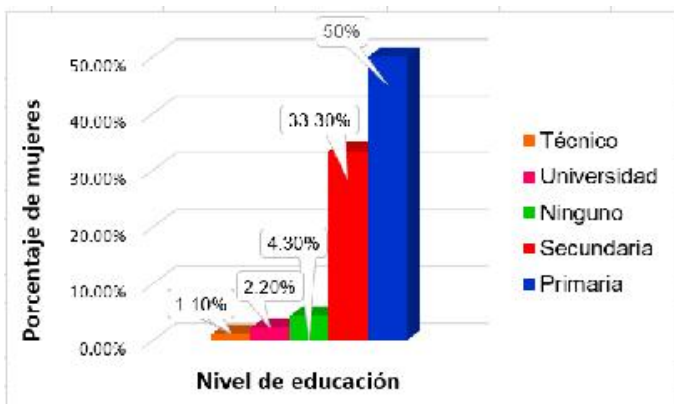

Figura 4.- Nivel de educación

En los últimos años en el Ecuador se han implementado sistemas educativos para erradicar el analfabetismo, sin embargo, no es suficiente para obtener un alto desarrollo de este grupo de mujeres. Se requiere de mayor capacitación técnica. El desarrollo técnico ayudara a mejorar las oportunidades de desarrollo de la calidad de vida del grupo de estudio (García, 2015).

Existencia de un contrato laboral. - Al respecto, la Figura 5 presenta cifras alarmantes: el $81 \%$ de mujeres involucradas con el sector agropecuario en el Cantón Rumiñahui no poseen ningún tipo de contrato con su empleador, y solamente un $19 \%$ indicó que si posee un contrato laboral.

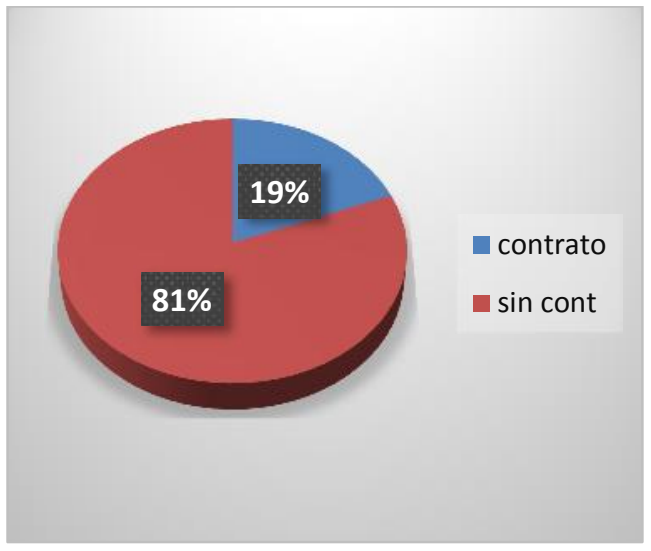

Figura 5.- existencia de un contrato laboral

En este aspecto es importante solucionar la falta de contratos para mejorar las condiciones laborales. La principal necesidad de las mujeres en este sector es formalizar su relación laboral y obtener garantía de empleo, estabilidad y remuneración fija, pues la falta de atención a estos factores, afectan directamente a la calidad de vida de las mujeres trabajadoras, la remuneración justa y adecuada es una de las más importantes junto con la salud y seguridad laboral.

Afiliación a algún seguro. - El 71\% de mujeres no poseen afiliación al seguro, por lo que carecen de un sistema de salud y seguridad ocupacional en el trabajo, también de otros beneficios que dota el Instituto Ecuatoriano de Seguridad Social $\mathrm{y}$ a pesar que trabajan la salud y seguridad ocupacional se convierte en una carga para el estado a través del Ministerio de Salud Pública. El 4,8\% posee afiliación al seguro campesino y el $24 \%$ posee afiliación al IESS tienen una gran ventaja para su calidad de vida en el trabajo.

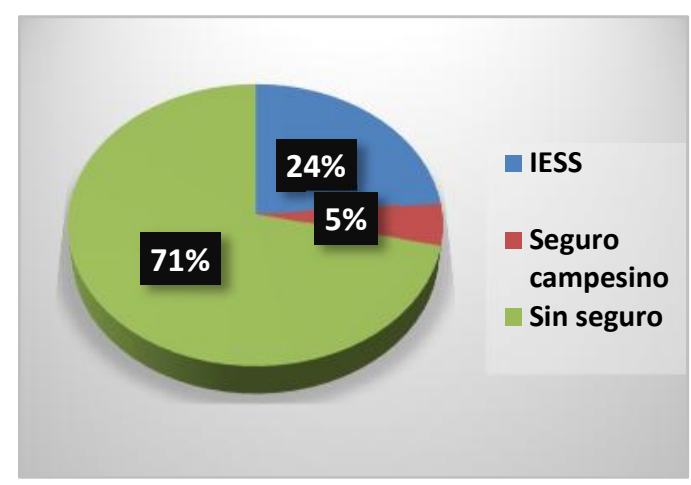

Figura 6.- Afiliación a algún seguro

Peligros expuestos en el trabajo.- Para este estudio se realizó la pregunta ¿Conoce usted los peligros a los que está expuesto en su trabajo?, en respuesta, el $20 \%$ de las mujeres trabajadoras en el sector agropecuario del Cantón Rumiñahui, desconoce los peligros a los que está expuesta en su trabajo, esto es grave porque en algunos casos aplican agroquímicos, fungicidas, etc., manejan productos veterinarios y expenden sus productos en el centro de distribución más importante de la zona (Figura 7).

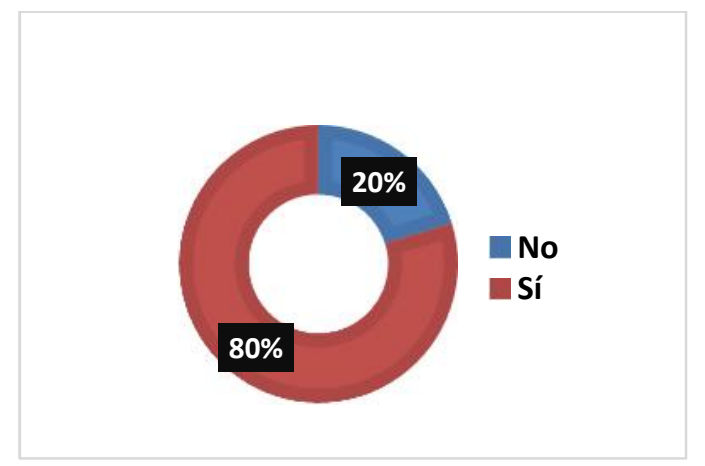

Figura 7.- Peligros expuestos en el trabajo 
Los resultados demuestran el nivel de conciencia de mujeres involucradas con el sector agropecuario en lo que respecta al conocimiento de los riesgos que corren en sus lugares de trabajo, mismos que se asocian con lo explicado por Domene (2012), para quien, existen varios riesgos laborales presentes en la actividad agropecuaria, tales como: riegos químicos (pueden romper la defensa que nos proporciona la piel y penetrar en el organismo. Irritaciones, quemaduras o úlceras, dermatitis, urticarias); riesgos mecánicos (las herramientas de trabajo son un peligro evidente. Cortes, laceraciones, aplastamiento o abrasión general, provocados por diferentes útiles de trabajo); riesgos térmicos (Asociado al calor o el frío y las inclemencias climatológicas. Quemaduras, debilitamiento de la capa córnea de la piel causan estrés térmico); riesgos eléctricos (Instalaciones eléctricas defectuosas o los cables mal aislados, equipamientos de trabajo, tareas de mantenimiento de la maquinaria podrían causar quemaduras y/o electrocución); riesgos producidos por vibración y movimientos repetitivos (maquinaria como tractores y equipos de trabajo empleados comúnmente y que forman parte del grupo de herramientas vibrátiles. Alteraciones circulatorias, dolor, incapacidad laboral causaría lesiones músculo-esqueléticas); otros riesgos como son los higiénicos y biológicos (Alergias provocadas por las materias. Dermatitis, urticarias, infecciones de las uñas, infecciones parasitarias. Mordedura-picadura de animales venenosos que incluye envenenamiento).

Tipos de peligros más frecuentes en el trabajo. - los principales riesgos a los que están expuestas las mujeres involucradas con el sector agropecuario en el Cantón Rumiñahui en su trabajo son, los riesgos físicos, riesgos químicos y riesgos psicosociales. Los riesgos nombrados con mayor frecuencia en la encuesta realizada son: la fumigación y exposición a agroquímicos, el exceso de carga de pesos, caídas, labores forzadas de campo, ataque de animales y como psicosocial el miedo a una erupción volcánica.

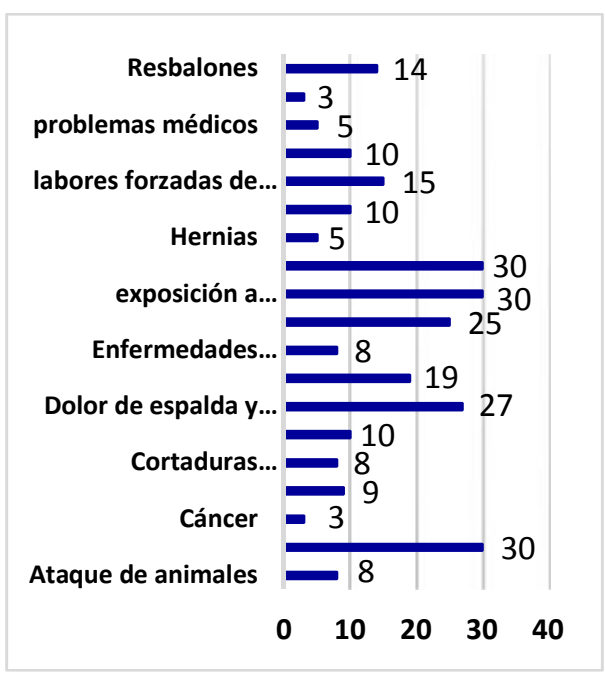

Figura 8.- Peligros expuestos en el trabajo

\section{Análisis de la fiabilidad de las dimensiones}

En el aplicativo IBM SPSS STADISTICS versión 2.2 se diseñó una base de datos, para tabular, organizar, sistematizar y analizar la información obtenida de la aplicación de la encuesta. Esta base de datos se organizó dividendo en las seis variables trazadoras. Salario o remuneración por el trabajo que realiza, Nivel de educación, Salario para satisfacer sus necesidades, Tipo de contrato, Afiliación algún tipo de seguro, Peligros a los que está expuesto en su trabajo. Cruzando con los valores de las cinco dimensiones.

Cuadro 3 - Análisis de fiabilidad de las dimensiones

\begin{tabular}{|l|l|l|}
\hline $\begin{array}{l}\text { Alfa de } \\
\text { Cron Bach }\end{array}$ & $\begin{array}{l}\text { Alfa de Cron Bach } \\
\text { basada en los } \\
\text { elementos } \\
\text { tipificados }\end{array}$ & $\begin{array}{l}\mathbf{N} \text { de } \\
\text { elementos }\end{array}$ \\
\hline, 859 &, 862 & 5 \\
\hline
\end{tabular}

El análisis de fiabilidad arrojó un valor de 0,862 que indica que tienen validez en la aplicación de la encuesta

Los resultados del "análisis de la varianza" OneWay ANOVA. El índice clave que se examina se conoce como prueba "F" (F-test) Después de realizar el análisis de datos, se obtiene que el valor del índice $\mathrm{F}(2,761)$ tiene un valor de $\mathrm{p}$ de 0,028 , que es menor de 0,5 por lo tanto es significativo. Esto quiere decir que existe relación entre las dimensiones y las variables que se miden en este estudio. La relación es significativa al obtener la percepción de las 
variables trazadoras con las dimensiones que miden la CVT del grupo de estudio.

\section{Pruebas de hipótesis para dimensiones de calidad de vida de trabajo}

Al ser comparados los datos con la información general. Los resultados de la prueba de $U$ de Mann-Whitney y la prueba de Kruskal Wallis, permiten conocer desde la estadística seis hipótesis para cada dimensión que son: salario o remuneración por el trabajo que realiza, nivel de educación, salario para satisfacer sus necesidades, tipo de contrato, afiliación algún tipo de seguro, peligros a los que está expuesto en su trabajo.

De la totalidad de la muestra evaluada para cada dimensión del instrumento CVT, se puede obtener el siguiente resumen e interpretación de los resultados de las relaciones que son estadísticamente representativas.

Aplicando la ecuación de contraste de hipótesis de Kruskal Wallis los datos resultaron estadísticamente representativos en la relación Seguridad en el trabajo con afiliación a algún tipo de seguro $\mathrm{P}=0,07 \leq 0,5$ al nivel de significancia. Aplicando la ecuación de contraste de hipótesis de U Mann Whitney los datos estadísticos que son representativos en la relación son: cantidad de remuneración adecuada para satisfacer las necesidades. En las dimensiones. Dimensión de Satisfacción por el trabajo el factor $\mathrm{p}=0,21 \leq 0,5$ al nivel de significancia; Dimensión de Seguridad en el trabajo el $p=0 \leq 0,5$ al nivel de significancia; Dimensión de bienestar logrado a través del trabajo $\mathrm{P}=0,01 \leq 0,5$ al nivel de significancia y para la Dimensión de desarrollo personal $\mathrm{P}=0 \leq 0,5$ al nivel de significancia

Se aprecia que las dimensiones de seguridad en el trabajo, bienestar logrado por el trabajo, administración del tiempo libre y satisfacción por el trabajo, se relacionan todas ellas con la cantidad de remuneración recibida por el trabajo, siendo la remuneración el factor de mayor importancia. Esto es comprensible teniendo en cuenta que un $69 \%$ de la muestra no recibe remuneración, es decir que si este factor mide la CVT, este grupo social se siente completamente insatisfecho con su calidad de vida.

Existe una limitada satisfacción por la modalidad de contratación, un $81 \%$ de la muestra no tiene contrato de trabajo por lo que esto genera inestabilidad laboral permanente. La afiliación a algún tipo de seguro es otro factor de limitada satisfacción, si bien por un lado tienen salud a través del ministerio de salud, por otra no poseen beneficios sociales como son: acceso a créditos de consumo, créditos hipotecarios u otros que otorga el IESS.

Respecto al desarrollo personal es la dimensión con mayor insatisfacción, esta dimensión valora la planificación del tiempo libre y el equilibrio entre el trabajo y la vida familiar, la falta de una remuneración junto con la deficiente educación son factores negativos por lo tanto no existe desarrollo personal en este grupo de estudio cuando sus condiciones de vida son precarias.

\section{Propuesta de mejora a las condiciones de calidad de vida en el trabajo de las mujeres del sector agropecuario en el Cantón Rumiñahui.}

Para el PUNTO CRÍTICO 1: Falta de tecnificación, capacitación en procesos de producción, y buenas prácticas de manufactura:

Objetivo: Crear alianzas estratégicas con centros de educación superior, Municipio de Rumiñahui, Consejo Provincial, Ministerio de Agricultura, para capacitaciones, orientadas al uso de tecnologías, uso racional de productos agroquímicos, manejo de suelos, agroindustria de productos, buenas prácticas de manufactura, comercio adecuado de productos perecibles, manejo de desechos entre otros, a fin de mejorar la calidad de vida de las mujeres, la calidad de los productos y del medio ambiente.

Estrategias a implementar: 1. Impulsar a las mujeres trabajadoras a participar en las capacitaciones técnicas que se impartirán por los organismos con el fin de mejorar la producción y calidad de los productos. 2. Enseñarles mediante capacitaciones el uso adecuado de plaguicidas, fungicidas, abonos, desinfectantes y coadyuvantes a fin de evitar la contaminación ambiental y proteger la salud pública. 3. Implementar una política $\mathrm{u}$ ordenanza municipal respecto el uso moderado de insumos agrícolas mediante el mapeo de distribuidores autorizados, asignación de presupuesto y capacitación técnica. 4. A través de capacitaciones mejorar la distribución de los productos, el empaque y dotar de cadenas de frio a los productos para la venta.

Metas e indicadores: 1. Incrementar el valor de los productos a través de la mejora de la calidad. 2. Mejorar el manejo de los productos, manejo de tiempos con una mejora de la economía financiera de las mujeres del sector.3. Remplazar los fertilizantes por el uso de nuevos tipos de abonos de tipo orgánico.

Alianzas Estratégicas: 1. Universidad de las Fuerzas Armadas ESPE (IASA). 2. Ministerio de agricultura y ganadería con el INIAP 


\section{Para el PUNTO CRÍTICO 2. Falta de formas de contratación, seguridad social, y seguridad laboral:}

Objetivo: Dotar de mejores condiciones a las mujeres trabajadoras del sector agropecuario mediante la afiliación al IESS, por parte del Municipio de Rumiñahui, convirtiéndose en empleador, de esta forma se les da garantía de empleo, salud laboral, seguridad laboral se mejoran las condiciones y se permite fortalecer su trabajo, prevenir el deterioro de la salud y la afectación por factores ambientales

Estrategias a implementar: 1. Afiliar a las mujeres trabajadoras al IESS, el patrono figurara como el Municipio de Rumiñahui. 2. Aplicar estrategias de posicionamiento en el mercado de los productos que producen. 3. Mejorar las condiciones de trabajo dotando de cadenas de frio, mejora de las cadenas de distribución y venta a los consumidores.

Metas e indicadores: Mejora de la calidad de vida mediante el acceso a salud, créditos quirografarios, créditos hipotecarios, entre otros, A través de las estrategias de posicionamiento se trabajará en productos, plaza, precio y publicidad y promoción, Al mejorar las condiciones de trabajo los productos mejoraran en Calidad y en precio.

Alianzas Estratégicas: Universidad de las Fuerzas Armadas ESPE, con el IASA, Ministerio de agricultura y ganadería con el INIAP

\section{Conclusiones}

La caracterización socio económica de las condiciones de calidad de vida del trabajo para las mujeres del sector agropecuario en el Cantón Rumiñahui, Provincia de Pichincha- Ecuador demostró condiciones de Calidad de Vida bajas, en razón de que no tienen acceso a capacitación agropecuaria, corren variados riesgos en su trabajo, no poseen seguridad laboral ni contratación que les dé estabilidad laboral, no todas tienen acceso a una paga justa por su trabajo, los productos agropecuarios que ellas producen no son valorados con un precio justo, $y$ consideran además que es necesario crear cadenas de valor para los productos. La intervención del estado mediante sus organismos como capacitador, formador de habilidades y destrezas, facilitador de créditos, fiscalizador de uso y aplicación de fertilizantes y pesticidas permitirá un desarrollo del sector mediante la utilización de nueva tecnología en la producción agropecuaria. El trabajo de la mujer en el campo garantiza el acceso a alimentos de calidad y orgánicos, es imperante mejorar las condiciones de salud y seguridad en el trabajo de estas, a más de una política pública referente a la mejora de sus ingresos.

\section{Referencias Bibliográficas}

Contreras, A. (2014). Chile en el Asia Pacífico. Las relaciones económicas con Vietnam, Cambodia y Laos en la última década. ISBN: 978-956-317-183-9.

Domene, M. 2012. Agricultura, una actividad sembrada de riesgos para las manos. Seguridad y Salud Laboral. Disponible en: http://archivosseguridadlaboralmanueldomene.blogspot.com/2012/01/agricultu ra-una-actividad-sembrada-de.html

García, S. 2015. Estudio de condiciones de calidad de vida en el trabajo de las mujeres en el sector agropecuario. Cantón Rumiñahui. Universidad Politécnica Nacional. QuitoEcuador. Tesis para optar por el grado de Magister en Gestión del Talento Humano. 128 p.

George, D. y Mallery, P. (2003). spss for Windows step by step: A Simple Guide and Reference. 11.0 Update (4. ${ }^{\mathrm{a}}$ ed.). Boston: Allyn \& Bacon.

Griffin, K. (2001). Desarrollo humano: origen, evolución e impacto. En P. Ibarra \& K. Unceta. Ensayos sobre el Desarrollo Humano (pág. 431). Barcelona, España: Icaria Editorial.

Instituto Nacional de estadísticas y Censos (INEC). 2010. Mujeres y hombres del Ecuador en cifras. III. Serie información estratégica. Editorial Ecuador. 184p. Disponible en: http://www.ecuadorencifras.gob.ec/wpcontent/descargas/Libros/Socioeconomico/Muje res_y_Hombres_del_Ecuador_en_Cifras_III.pdf

Instituto Nacional de estadísticas y Censos (INEC). 2016. Índice de Producción Industrial Índice de Empleo, Remuneraciones y Horas Trabajadas. Disponible en: http://www.ecuadorencifras.gob.ec//documentos /web-inec/Estadisticas_Economicas/IERIVI/Publicaciones_IER/IER 2016/IPIIER_PUBLICACION_ENE_2016.pdf

Keith, G. (2001). Desarrollo Humano: Origen, evolución e impacto. Obtenido de Griffin, K. (2001). Desarrollo humano: origen, evolución e impacto. Ensayos sobre el desarrollo humano, 25-40.

Osorio, V. (07 de 03 de 2014). Colectivo de abogados. Obtenido de colectivo de abogados: http://www.colectivodeabogados.org/informes/i nformes-nacionales/La-situacion-laboral-de-las 
Peñarrieta, I; Abregú, S; Krederdt, S; Guevara, G; Carhuapoma, M; Chavez, F. 2014. "Validación del instrumento: "Calidad de vida en el trabajo "CVT-GOHISALO"en enfermería del primer nivel de atención". Rev enferm Herediana: 7(2):124-131.

Pérez, J; Navarrete, D; García, R. 2011. Perspectiva Organizacional y Psicosocial de los Efectos de las Organizaciones en la Salud Ocupacional del Factor Humano. XV Congreso Internacional de Investigación en Ciencias
Administrativas. Capítulo 17- Teorías de la Organización. 23-36 pág. http://acacia.org. mx/busqueda/pdf/17_01_perspectiva_organizaci onal.pdf

Pérez, J. P. (2013). Efecto del burnout y la sobrecarga en la calidad de vida en el trabajo. elsevier, Volume 29, Issue 129, Pages 445-455.

Reyes, G. 2009. Teorías de Desarrollo Económico y Social: Articulación con el Planteamiento de Desarrollo Humano. Tendencias. Revista de la Facultad de Ciencias Económicas y Administrativas Universidad de Nariño. 1: 117-142.

Sen A. (1992), Nuevo examen de la desigualdad, Madrid: Alianza.

Welch, S. y Comer, J. (1988). Quantitative Methods for Public Administration: Techniques And Applications. Editorial Books/Cole Publishing Co. ISBN 10:O534108881/ 13: 9780534108885. U.S. 